Cochran, J. R., Stow, D.A.V., et al., 1990

Proceedings of the Ocean Drilling Program, Scientific Results, Vol. 116

\title{
33. GRAIN-SIZE ANALYSIS: LEG 116, BENGAL FAN ${ }^{1}$
}

\author{
Peter S. Balson ${ }^{2}$ and Dorrik A. V. Stow ${ }^{3,4}$
}

\section{INTRODUCTION}

Ocean Drilling Program (ODP) Leg 116 cored the distal part of the Bengal Fan at three closely spaced sites (717-719). The recovered sediments consisted dominantly of turbidites that varied in thickness between a few centimeters and $2 \mathrm{~m}$ or more. A number of different facies have been identified in the sequence and are described by Stow et al. (this volume). Representative examples of these facies types were selected and sampled for grain-size analysis. The results of these analyses are tabulated in this data report.

\section{METHODS}

Sediment samples of approximately 2 -g dry weight were placed in $50-\mathrm{cm}^{3}$ beakers and disaggregated in a solution of $10 \%$ Calgon. To assist in complete disaggregation of the sediment, an ultrasonic probe was used for approximately 2 min. on each sample. Suspended sample concentrations were of the order of $20 \mathrm{~g} / \mathrm{L}$. The prepared suspensions were stored in $150-\mathrm{cm}^{3}$ plastic bottles.

The grain-size analyses were performed by laser diffraction using Malvern particle sizers, models $3500 \mathrm{D}$ and $2600 \mathrm{C}$. The principles of particle-size analysis by laser diffraction are described by Butters and Wheatley (1982) and McCave et al. (1986). Laser diffraction is a sizing technique in which the degree of scattering of a laser beam reflects the size of the grains that pass through the beam. The data obtained is therefore quite different from that derived from settling techniques such as the Andreason pipette and Sedigraph.

The Malvern laser-sizers consist of a laser source, beam expander, sample chamber, focusing lens, ring detector, and a microcomputer. In this study each analysis used a $100-\mathrm{mm}$ focal length focusing lens yielding data consisting of 15 size classes between 1.9 and $188 \mu \mathrm{m}$. A percentage of sample outside of this range above and below these limits is also given. The grain-size distribution was computed using the model-independent program option. The sample suspension is introduced into a small ultrasonic tank from which the suspension is continuously pumped through the sample chamber in the path of the laser. The main attraction of laser diffraction analysis for this study was the relatively small sample size required for analysis. Only $2-5 \mathrm{~cm}^{3}$ of the prepared suspensions are required for each analysis. Several repeat analyses can therefore be performed even on very small original samples. An additional advantage is the speed with which analyses can be performed, generally about $10 \mathrm{~min}$ per sample.

For many of the samples, duplicate or triplicate analyses were performed to test the reproducibility of the results.

\footnotetext{
${ }^{1}$ Cochran, J. R., Stow, D.A.V., et al., 1990. Proc. ODP, Sci. Results, 116: College Station, TX (Ocean Drilling Program).

2 British Geological Survey, Keyworth, Nottingham, NG12 5GG, U.K.

${ }^{3}$ University of Nottingham, Nottingham, NG7 2RD, U.K.

${ }^{4}$ Present address: Geology Department, The University, Southampton, S09 5NH, U.K.
}

Results proved to be very reproducible both for repeated analyses of the same sample and for multiple subsamples, which is in accordance with the findings of Butters and Wheatley (1982), and McCave et al. (1986). Butters and Wheatley (1982) found an acceptable level of agreement between particle-size analyses of quartz standards obtained with a Malvern laser-sizer and standard certified data. Some authors, however, have found deficiencies in laser-sizers, especially where large quantities of fine material are present e.g., McCave et al. (1986) and Singer et al. (1988), in particular a tendency to underestimate the quantity of clay $(<1.9 \mu \mathrm{m}$ in those studies) present. The data presented herein should be viewed with this possible limitation in mind.

\section{RESULTS}

A total of 139 samples have been analyzed and the results tabulated in Tables 1,2 , and 3. The mean $\left(M_{z}\right)$, sorting $\left(\sigma_{i}\right)$ and skewness $\left(S k_{i}\right)$ were calculated for all samples according to methods of Folk and Ward (1957). The lithological symbols used relate to the classification of Shepard (1954) and are an extended form of symbols used in the Leg 116 shipboard descriptions (See Fig. 1).

The results include spot samples from a variety of sediment facies in Site 717 holes (Samples 1-42). Suites of samples taken from selected individual turbidites in Sites 717, 718, and 719 holes (Samples 121-210) and a single suite of eight closely spaced samples (Samples 43-50) were used to test grain-size variations over a short vertical sequence $(16 \mathrm{~cm})$ within a thick turbidite unit. They also were used to examine the scatter of results to be expected from the analytical method. The results for Samples 43-50 show that grain-size characteristics are very consistent over a short vertical sequence, and no clear trends are visible. This conclusion is supported by the more widely spaced data points represented by Samples 121-210, which show smooth trends with very little "noise," that might be attributed to the analytical method. The grain-size characteristics of the turbidite facies are discussed in the sediment synthesis paper by Stow et al. (this volume). The sediment facies from which samples were taken are given in Tables 1-3. These are as follows:

Facies 1: light gray silt and mud turbidites

Facies 2: light gray organic-poor mud turbidites

Facies 3: dark gray organic-rich mud turbidites

Facies 4: biogenic mud turbidites

Facies 5: pelagic clays

Facies 6: pelagic calcareous clays

Facies 7: structureless bioturbated muds

\section{ACKNOWLEDGMENTS}

We are grateful to the University of Nottingham and British Gypsum Ltd. for access to laser size-analyzers for this study and to S. Wright and J. Karkut for technical assistance. Published with the permission of the Director, British Geological Survey (N.E.R.C.). 
Table 1. Hole 717C grain-size analysis data.

\begin{tabular}{|c|c|c|c|c|c|c|c|c|c|c|c|}
\hline No. & Core & Sect. & $\begin{array}{l}\text { Depth } \\
\text { (cm) }\end{array}$ & $\begin{array}{c}\text { Sand } \\
(\%)\end{array}$ & $\begin{array}{l}\text { Silt } \\
(\%)\end{array}$ & $\begin{array}{l}\text { Clay } \\
(\%)\end{array}$ & $\begin{array}{l}M_{2} \\
(\phi)\end{array}$ & $\begin{array}{c}\sigma_{i} \\
(\phi)\end{array}$ & $S k_{i}$ & Lith. & Facies \\
\hline 1 & $2 \mathrm{H}$ & 2 & $85-90$ & 47 & 50 & 3 & 4.32 & 1.50 & 0.32 & T7B & 1 \\
\hline 2 & $14 \mathrm{X}$ & 2 & $8-13$ & 0 & 78 & 22 & 7.01 & 0.99 & -0.03 & T5 & 1 \\
\hline 3 & $19 X$ & 1 & $40-45$ & 13 & 76 & 11 & 5.85 & 1.61 & 0.01 & T5 & 1 \\
\hline 4 & $20 \mathrm{X}$ & 1 & $80-85$ & 24 & 70 & 6 & 5.16 & 1.60 & 0.13 & T7B & 1 \\
\hline 5 & $20 X$ & 3 & $50-55$ & 0 & 72 & 28 & 7.24 & 0.88 & -0.03 & T8A & 2 \\
\hline 6 & $21 X$ & 2 & $138-143$ & 0 & 60 & 40 & 7.55 & 0.86 & -0.17 & T8A & 4 \\
\hline 7 & $22 \mathrm{X}$ & $\mathrm{cc}$ & $28-30$ & 0 & 52 & 48 & 7.78 & 0.78 & -0.08 & T8A & 4 \\
\hline 8 & $26 \mathrm{X}$ & 4 & $80-85$ & 8 & 77 & 15 & 6.03 & 1.46 & 0.19 & T5 & 1 \\
\hline 9 & $29 X$ & 2 & $83-88$ & 0 & 67 & 33 & 7.14 & 1.03 & 0.04 & $\mathrm{~T} 8 \mathrm{~A}$ & 3 \\
\hline 10 & $31 X$ & 2 & $100-105$ & 0 & 73 & 27 & 6.86 & 1.17 & 0.10 & T8A & 3 \\
\hline 11 & $31 X$ & 2 & $132-137$ & 0 & 62 & 38 & 7.39 & 0.98 & -0.09 & T8A & 3 \\
\hline 12 & $34 X$ & 2 & $80-85$ & 0 & 71 & 29 & 7.05 & 1.10 & 0.01 & T8A & 4 \\
\hline 13 & $36 \mathrm{X}$ & 2 & $82-87$ & 0 & 53 & 47 & 7.76 & 0.77 & -0.07 & T8A & 3 \\
\hline 14 & $37 X$ & 1 & $82-87$ & 16 & 80 & 4 & 5.21 & 1.37 & 0.15 & T5 & 1 \\
\hline 15 & $38 \mathrm{X}$ & 2 & $85-90$ & 0 & 81 & 19 & 6.96 & 0.93 & 0.07 & T5 & 2 \\
\hline 16 & $40 X$ & 1 & $80-85$ & 0 & 69 & 31 & 7.38 & 0.75 & -0.10 & T8A & 3 \\
\hline 17 & $40 X$ & 3 & $115-120$ & 0 & 47 & 53 & 7.95 & 0.64 & 0.15 & T8B & 4 \\
\hline 18 & $42 X$ & 2 & $98-103$ & 0 & 74 & 26 & 7.71 & 1.01 & -0.07 & T8A & 2 \\
\hline 19 & $43 X$ & 5 & $80-85$ & 0 & 55 & 45 & 7.58 & 0.94 & -0.16 & T8A & 3 \\
\hline 20 & $44 X$ & 2 & $53-58$ & 0 & 69 & 31 & 7.28 & 1.02 & -0.11 & $\mathrm{~T} 8 \mathrm{~A}$ & 4 \\
\hline 21 & $45 X$ & 6 & $73-78$ & 0 & 71 & 29 & 7.12 & 1.07 & -0.05 & T8A & 3 \\
\hline 22 & $46 X$ & 2 & $56-61$ & 0 & 67 & 33 & 7.38 & 0.93 & -0.09 & T8A & 7 \\
\hline 23 & $47 X$ & 2 & $113-118$ & 0 & 70 & 30 & 7.31 & 0.93 & -0.05 & T8A & 2 \\
\hline 24 & $48 X$ & 2 & $83-88$ & 0 & 57 & 43 & 7.41 & 1.02 & -0.05 & T8A & 3 \\
\hline 26 & $51 X$ & 3 & $12-17$ & 0.4 & 75.2 & 24.4 & 6.53 & 1.30 & 0.14 & T5 & 3 \\
\hline 27 & $53 \mathrm{X}$ & 2 & $80-85$ & 0 & 77 & 23 & 7.05 & 1.02 & -0.03 & T5 & 2 \\
\hline 28 & $53 \mathrm{X}$ & 5 & $80-84$ & 1 & 89 & 10 & 6.13 & 1.19 & 0.16 & T5 & 1 \\
\hline 29 & $54 \mathrm{X}$ & 2 & $80-85$ & 0 & 77 & 23 & 7.00 & 1.04 & -0.02 & T5 & 2 \\
\hline 30 & $56 \mathrm{X}$ & 4 & $78-83$ & 0 & 67 & 33 & 7.37 & 0.95 & -0.08 & T8A & 7 \\
\hline 31 & $57 \mathrm{X}$ & 5 & $80-85$ & 0 & 43 & 57 & 7.90 & 0.78 & -0.16 & T8B & 5 \\
\hline 32 & $59 \mathrm{X}$ & 1 & $27-32$ & 1 & 87 & 12 & 6.35 & 1.21 & 0.13 & T5 & 1 \\
\hline 33 & $61 X$ & 2 & $85-90$ & 1 & 86 & 13 & 6.44 & 1.27 & -0.02 & T5 & $i$ \\
\hline 34 & $64 X$ & 3 & $90-95$ & 0 & 70 & 30 & 7.32 & 0.94 & -0.07 & T8A & 2 \\
\hline 35 & $65 x$ & 3 & $30-37$ & 0 & 69 & 31 & 7.02 & 0.97 & 0.08 & T8A & 3 \\
\hline 36 & $65 X$ & 3 & $52-57$ & 0 & 75 & 25 & 7.21 & 0.93 & 0.00 & T5 & 3 \\
\hline 37 & $65 \mathrm{X}$ & 3 & $117-122$ & 0.4 & 74.5 & 25.1 & 6.62 & 1.33 & 0.06 & T8A & 3 \\
\hline 38 & $68 \mathrm{X}$ & 2 & $70-75$ & 0 & 62 & 38 & 7.47 & 0.87 & -0.11 & $\mathrm{~T} 8 \mathrm{~A}$ & 2 \\
\hline 39 & $70 x$ & 6 & $7-12$ & 0 & 75 & 25 & 7.15 & 0.98 & -0.03 & T5 & 7 \\
\hline 40 & $71 X$ & 2 & $29-34$ & 0 & 82 & 18 & 6.85 & 1.04 & 0.03 & T5 & 1 \\
\hline 41 & $72 X$ & 2 & $75-80$ & 0 & 77 & 23 & 7.05 & 0.98 & 0.01 & T5 & 2 \\
\hline 42 & $74 X$ & 1 & $80-84$ & 12 & 80 & 8 & 5.69 & 1.53 & 0.04 & T5 & 1 \\
\hline 43 & $22 \mathrm{X}$ & 4 & $134-136$ & 0 & 57 & 43 & 7.65 & 0.87 & -0.16 & T8A & 2 \\
\hline 44 & $22 X$ & 4 & $136-138$ & 0 & 51 & 49 & 7.84 & 0.69 & -0.02 & T8A & 2 \\
\hline 45 & $22 \mathrm{X}$ & 4 & $138-140$ & 0 & 52 & 48 & 7.79 & 0.75 & -0.06 & T8A & 2 \\
\hline 46 & $22 X$ & 4 & $140-142$ & 0 & 54 & 46 & 7.77 & 0.76 & -0.05 & T8A & 2 \\
\hline 47 & $22 \mathrm{X}$ & 4 & $142-144$ & 0 & 55 & 45 & 7.77 & 0.75 & -0.07 & T8A & 2 \\
\hline 48 & $22 X$ & 4 & $144-146$ & 0 & 53 & 47 & 7.77 & 0.76 & -0.06 & T8A & 2 \\
\hline 49 & $22 X$ & 4 & $146-148$ & 0 & 55 & 45 & 7.75 & 0.77 & -0.10 & T8A & 2 \\
\hline 50 & $22 \mathrm{X}$ & 4 & $148-150$ & 0 & 52 & 48 & 7.80 & 0.75 & -0.05 & T8A & 2 \\
\hline 121 & $2 \mathrm{H}$ & 4 & $2-3$ & 0 & 69 & 31 & 7.28 & 0.84 & -0.16 & T8A & 6 \\
\hline 122 & $2 \mathrm{H}$ & 4 & $11-12$ & 5 & 83 & 12 & 6.20 & 1.41 & 0.00 & T5 & 1 \\
\hline 123 & $2 \mathrm{H}$ & 4 & $25-26$ & 9 & 86 & 5 & 5.59 & 1.32 & 0.15 & T5 & $i$ \\
\hline 124 & $2 \mathrm{H}$ & 4 & $51-52$ & 13 & 83 & 4 & 5.21 & 1.25 & 0.25 & T5 & 1 \\
\hline 125 & $2 \mathrm{H}$ & 4 & $100-101$ & 29 & 68 & 3 & 4.88 & 1.44 & 0.16 & $\mathrm{~T} 7 \mathrm{~B}$ & $i$ \\
\hline 126 & $2 \mathrm{H}$ & 4 & $140-141$ & 39 & 58 & 3 & 4.60 & 1.42 & 0.17 & $\mathrm{~T} 7 \mathrm{~B}$ & 1 \\
\hline 127 & $2 \mathrm{H}$ & 5 & $14-15$ & 29 & 68 & 3 & 4.81 & 1.44 & 0.13 & T7B & $i$ \\
\hline 128 & $23 x$ & 2 & $20-21$ & 0 & 64 & 36 & 7.43 & 0.94 & -0.13 & T8A & $5 / 6$ \\
\hline 129 & $23 X$ & 2 & $50-51$ & 0.3 & 65.1 & 34.6 & 7.17 & 1.19 & -0.13 & T8A & 4 \\
\hline 130 & $23 \mathrm{X}$ & 2 & $100-101$ & 1 & 66 & 33 & 6.95 & 1.41 & -0.16 & T8A & 4 \\
\hline 131 & $23 \mathrm{X}$ & 2 & $140-141$ & 7 & 69 & 24 & 6.43 & 1.64 & -0.13 & T8A & 4 \\
\hline 132 & $23 \mathrm{X}$ & 3 & $6-7$ & 20 & 67 & 13 & 5.52 & 1.72 & 0.22 & $\mathrm{~T} 7 \mathrm{~B}$ & 4 \\
\hline 133 & $23 \mathrm{X}$ & 3 & $15-16$ & 0 & 49 & 51 & 7.86 & 0.72 & -0.04 & T8B & 5 \\
\hline 134 & $23 X$ & 3 & $24-25$ & 0 & 59 & 41 & 7.63 & 0.81 & -0.21 & T8A & 3 \\
\hline 135 & $23 x$ & 3 & $40-41$ & 0 & 63 & 37 & 7.38 & 0.97 & -0.06 & T8A & 3 \\
\hline 136 & $23 X$ & 3 & $66-67$ & 0 & 64 & 36 & 7.29 & 1.07 & -0.07 & T8A & 3 \\
\hline 137 & $23 x$ & 3 & $87-88$ & 0 & 68 & 32 & 7.13 & 1.12 & -0.03 & T8A & 3 \\
\hline 138 & $23 X$ & 3 & $96-97$ & 1 & 82 & 17 & 6.39 & 1.27 & 0.12 & T5 & 3 \\
\hline 139 & $33 X$ & 5 & $26-27$ & 0 & 56 & 44 & 7.68 & 0.79 & 0.06 & T8A & $5 / 7$ \\
\hline 140 & $33 x$ & 5 & $39-40$ & 0 & 65 & 35 & 7.42 & 0.82 & 0.00 & T8A & 3 \\
\hline 141 & $33 X$ & 5 & $56-57$ & 0 & 38 & 62 & 7.98 & 0.67 & -0.20 & T8B & 3 \\
\hline 142 & $33 x$ & 5 & $70-71$ & 0 & 52 & 48 & 7.73 & 0.79 & 0.05 & $\mathrm{~T} 8 \mathrm{~A}$ & 3 \\
\hline 143 & $33 X$ & 5 & $86-87$ & 0 & 51 & 49 & 7.76 & 0.75 & 0.09 & $\mathrm{~T} 8 \mathrm{~A}$ & 3 \\
\hline 144 & $36 \mathrm{X}$ & 2 & $75-76$ & 0 & 50 & 50 & 7.79 & 0.74 & 0.07 & T8AB & 7 \\
\hline 145 & $36 X$ & 2 & $100-101$ & 0 & 54 & 46 & 7.70 & 0.79 & 0.08 & T8A & 3 \\
\hline 146 & $36 X$ & 2 & $135-136$ & 0 & 54 & 46 & 7.70 & 0.79 & 0.12 & T8A & 3 \\
\hline 147 & $36 \mathrm{X}$ & 3 & $1-2$ & 0 & 57 & 43 & 7.62 & 0.84 & 0.10 & T8A & 3 \\
\hline 148 & $36 \mathrm{X}$ & 3 & $11-12$ & 0 & 46 & 54 & 7.84 & 0.75 & 0.08 & T8B & 7 \\
\hline
\end{tabular}


Table 1 (continued).

\begin{tabular}{llllllllllll}
\hline No. & Core & Sect. & $\begin{array}{c}\text { Depth } \\
(\mathrm{cm})\end{array}$ & $\begin{array}{c}\text { Sand } \\
(\%)\end{array}$ & $\begin{array}{c}\text { Silt } \\
(\%)\end{array}$ & $\begin{array}{c}\text { Clay } \\
(\%)\end{array}$ & $\begin{array}{c}M_{2} \\
(\phi)\end{array}$ & $\begin{array}{c}\sigma_{i} \\
(\phi)\end{array}$ & \multicolumn{1}{c}{$S k_{i}$} & Lith. & Facies \\
\hline 149 & $36 \mathrm{X}$ & 3 & $33-34$ & 0 & 57 & 43 & 7.61 & 0.84 & 0.10 & T8A & 3 \\
150 & $36 \mathrm{X}$ & 3 & $55-56$ & 0 & 70 & 30 & 7.26 & 0.98 & 0.08 & T8A & 3 \\
151 & $36 \mathrm{X}$ & 3 & $85-86$ & 0 & 72 & 28 & 7.13 & 1.01 & 0.06 & T8A & 3 \\
152 & $36 \mathrm{X}$ & 3 & $115-116$ & 0.3 & 77.6 & 22.1 & 6.72 & 1.17 & 0.04 & T5 & 3 \\
153 & $36 \mathrm{X}$ & 3 & $131-132$ & 0.7 & 87.8 & 11.5 & 5.99 & 1.22 & 0.28 & T5 & 3 \\
154 & $36 \mathrm{X}$ & 3 & $136-137$ & 0 & 59 & 41 & 7.66 & 0.78 & 0.09 & T8A & 5 \\
155 & $48 \mathrm{X}$ & 2 & $55-56$ & 0 & 50 & 50 & 7.78 & 0.75 & 0.15 & T8AB & 7 \\
156 & $48 \mathrm{X}$ & 2 & $77-78$ & 0 & 44 & 56 & 7.87 & 0.75 & -0.03 & T8B & 3 \\
157 & $48 \mathrm{X}$ & 2 & $94-95$ & 0 & 58 & 42 & 7.59 & 0.86 & 0.09 & T8A & 3 \\
158 & $48 \mathrm{X}$ & 2 & $112-113$ & 0 & 62 & 38 & 7.45 & 0.95 & 0.08 & T8A & 3 \\
159 & $48 \mathrm{X}$ & 2 & $134-135$ & 0 & 61 & 39 & 7.42 & 1.00 & 0.02 & T8A & 3 \\
160 & $48 \mathrm{X}$ & 3 & $24-25$ & 0.6 & 81.4 & 18.0 & 6.29 & 1.30 & 0.25 & T5 & 3 \\
161 & $48 \mathrm{X}$ & 3 & $33-34$ & 0 & 63 & 37 & 7.54 & 0.82 & 0.10 & T8A & 5 \\
162 & $48 \mathrm{X}$ & 3 & $44-45$ & 0 & 58 & 42 & 7.66 & 0.80 & 0.11 & T8A & 5 \\
163 & $48 \mathrm{X}$ & 3 & $64-65$ & 0 & 69 & 31 & 7.33 & 0.87 & 0.00 & T8A & 2 \\
164 & $48 \mathrm{X}$ & 3 & $84-85$ & 0 & 70 & 30 & 7.28 & 0.90 & 0.00 & T8A & 2 \\
165 & $48 \mathrm{X}$ & 3 & $103-104$ & 0 & 74 & 26 & 7.01 & 1.01 & 0.04 & T8A & 2 \\
166 & $68 \mathrm{X}$ & 1 & $63-64$ & 0 & 78 & 22 & 7.14 & 0.86 & 0.05 & T5 & 1 \\
167 & $68 \mathrm{X}$ & 1 & $64-65$ & 0.2 & 88.7 & 11.1 & 6.56 & 0.98 & 0.13 & T5 & 1 \\
168 & $68 \mathrm{X}$ & 1 & $67-68$ & 0 & 82 & 18 & 7.11 & 0.91 & 0.02 & T5 & 1 \\
169 & $68 \mathrm{X}$ & 1 & $71-72$ & 0.1 & 89.8 & 10.1 & 6.53 & 1.05 & 0.07 & T5 & 1 \\
170 & $68 \mathrm{X}$ & 1 & $75-76$ & 1.4 & 86.0 & 12.6 & 6.27 & 1.30 & 0.08 & T5 & 1 \\
171 & $68 \mathrm{X}$ & 1 & $80-81$ & 0 & 64 & 36 & 7.46 & 0.92 & -0.10 & T8A & 5 \\
172 & $68 \mathrm{X}$ & 1 & $93-94$ & 0 & 64 & 36 & 7.50 & 0.88 & -0.08 & T8A & 1 \\
173 & $68 \mathrm{X}$ & 1 & $104-105$ & 0 & 83 & 17 & 6.88 & 0.99 & 0.06 & T5 & 1 \\
174 & $68 \mathrm{X}$ & 1 & $115-116$ & 13 & 82 & 5 & 5.53 & 1.42 & 0.10 & T5 & 1 \\
175 & $68 \mathrm{X}$ & 1 & $129-130$ & 11 & 83 & 6 & 5.64 & 1.40 & 0.11 & T5 & 1 \\
\hline & & & & & & & & & & &
\end{tabular}

Table 2. Holes 718A, 718B, 718C, and 718D grain-size analysis data.

\begin{tabular}{|c|c|c|c|c|c|c|c|c|c|c|c|}
\hline No. & Core & Sect. & $\begin{array}{c}\text { Depth } \\
(\mathrm{cm})\end{array}$ & $\begin{array}{c}\text { Sand } \\
(\%)\end{array}$ & $\begin{array}{l}\text { Silt } \\
(\%)\end{array}$ & $\begin{array}{c}\text { Clay } \\
(\%)\end{array}$ & $\begin{array}{l}M_{z} \\
(\phi)\end{array}$ & $\begin{array}{c}\sigma_{i} \\
(\phi)\end{array}$ & $S k_{i}$ & Lith. & Facies \\
\hline 176 & $17 X$ & 5 & $6-7$ & 0 & 73 & 27 & 7.25 & 0.93 & -0.04 & T8A & 6 \\
\hline 177 & $17 X$ & 5 & $12-13$ & 0 & 76 & 24 & 7.15 & 0.96 & -0.03 & T5 & 7 \\
\hline 178 & $17 X$ & 5 & $18-19$ & 0 & 60 & 40 & 7.44 & 0.94 & -0.06 & T8A & 7 \\
\hline 179 & $17 X$ & 5 & $25-26$ & 0 & 69 & 31 & 7.10 & 1.09 & -0.03 & T8A & 7 \\
\hline 180 & $17 X$ & 5 & $26-27$ & 0 & 62 & 38 & 7.40 & 0.98 & -0.06 & T8A & 7 \\
\hline 181 & $17 X$ & 5 & $33-34$ & 0 & 59 & 41 & 7.46 & 0.97 & -0.04 & T8A & 7 \\
\hline 182 & $17 X$ & 5 & $40-41$ & 0 & 69 & 31 & 7.12 & 1.04 & 0.02 & T8A & 3 \\
\hline 183 & $17 X$ & 5 & $42-43$ & 0 & 64 & 36 & 7.38 & 0.91 & -0.08 & T8A & 3 \\
\hline 184 & $17 X$ & 5 & $64-65$ & 0 & 64 & 36 & 7.32 & 0.94 & -0.08 & T8A & 2 \\
\hline 185 & $17 X$ & 5 & $79-80$ & 0 & 70 & 30 & 7.30 & 0.95 & -0.06 & T8A & 2 \\
\hline 186 & $17 X$ & 5 & $88-89$ & 0 & 72 & 28 & 7.23 & 0.98 & -0.05 & T8A & 2 \\
\hline 187 & $17 \mathrm{X}$ & 5 & 96-97 & 0.3 & 90.3 & 9.4 & 6.31 & 1.11 & 0.14 & T5 & 2 \\
\hline 188 & $17 \mathrm{X}$ & 5 & $116-117$ & 0 & 68 & 32 & 7.22 & 1.03 & -0.04 & T8A & 3 \\
\hline 189 & $17 X$ & 5 & $126-127$ & 0 & 62 & 38 & 7.39 & 0.97 & -0.05 & T8A & 3 \\
\hline 190 & $17 X$ & 5 & $133-134$ & 0 & 83 & 17 & 6.58 & 1.08 & 0.21 & T5 & 3 \\
\hline 191 & $52 \mathrm{X}$ & 1 & $12-13$ & 3 & 89 & 8 & 5.84 & 1.37 & 0.10 & T5 & 1 \\
\hline 192 & $52 \mathrm{X}$ & 1 & $37-38$ & 5 & 83 & 12 & 6.02 & 1.42 & 0.11 & T5 & 1 \\
\hline 193 & $52 \mathrm{X}$ & 1 & $69-70$ & 16 & 79 & 5 & 5.29 & 1.42 & 0.20 & T7B & 1 \\
\hline 194 & $52 \mathrm{X}$ & 1 & $96-97$ & 0.3 & 85.5 & 14.2 & 6.52 & 1.21 & 0.03 & T5 & 1 \\
\hline 195 & $52 \mathrm{X}$ & 1 & $123-124$ & 2 & 85 & 13 & 6.22 & 1.34 & 0.08 & T5 & 1 \\
\hline 196 & $94 X$ & 5 & $36-37$ & 0 & 61 & 39 & 7.51 & 0.85 & -0.14 & T8A & 5 \\
\hline 197 & $94 X$ & 5 & $40-41$ & 0 & 83 & 17 & 6.86 & 1.01 & 0.05 & T5 & 2 \\
\hline 198 & $94 X$ & 5 & $43-44$ & 0 & 87 & 13 & 6.72 & 0.99 & 0.07 & T5 & 2 \\
\hline 199 & $94 X$ & 5 & $136-137$ & 0 & 60 & 40 & 7.51 & 0.87 & -0.14 & T8A & 5 \\
\hline 200 & $94 X$ & 5 & $139-140$ & 0 & 62 & 38 & 7.48 & 0.86 & -0.11 & T8A & 2 \\
\hline 201 & $94 \mathrm{X}$ & 5 & $141-142$ & 0 & 68 & 32 & 7.38 & 0.92 & -0.05 & T8A & 2 \\
\hline
\end{tabular}


Table 3. Holes 719A and 719B grain-size analysis data.

\begin{tabular}{cccccccccccc}
\hline No. & Core & Sect. & $\begin{array}{c}\text { Depth } \\
(\mathrm{cm})\end{array}$ & $\begin{array}{c}\text { Sand } \\
(\%)\end{array}$ & $\begin{array}{c}\text { Silt } \\
(\%)\end{array}$ & $\begin{array}{c}\text { Clay } \\
(\%)\end{array}$ & $\begin{array}{c}M_{z} \\
(\phi)\end{array}$ & $\begin{array}{c}\sigma_{i} \\
(\phi)\end{array}$ & $S k_{i}$ & Lith. & Facies \\
\hline 202 & $18 \mathrm{X}$ & 2 & $27-28$ & 0 & 59 & 41 & 7.44 & 0.99 & -0.09 & T8A & 7 \\
203 & $18 \mathrm{X}$ & 2 & $37-38$ & 0 & 61 & 39 & 7.42 & 0.99 & -0.09 & T8A & 7 \\
204 & $18 \mathrm{X}$ & 2 & $50-51$ & 0 & 66 & 34 & 7.36 & 0.96 & -0.06 & T8A & 7 \\
205 & $18 \mathrm{X}$ & 2 & $60-61$ & 0 & 58 & 42 & 7.54 & 0.91 & -0.02 & T8A & 7 \\
206 & $18 \mathrm{X}$ & 2 & $64-65$ & 0 & 58 & 42 & 7.53 & 0.91 & -0.03 & T8A & $3 / 7$ \\
207 & $18 \mathrm{X}$ & 3 & $35-36$ & 0 & 74 & 26 & 7.14 & 1.00 & -0.01 & T8A & $2 / 7$ \\
208 & $18 \mathrm{X}$ & 3 & $22-23$ & 0 & 74 & 26 & 7.10 & 1.03 & -0.03 & T8A & $2 / 7$ \\
209 & $18 \mathrm{X}$ & 3 & $44-45$ & 0 & 77 & 23 & 6.96 & 1.07 & 0.01 & T5 & $2 / 7$ \\
210 & $18 \mathrm{X}$ & 3 & $54-55$ & 0.5 & 86.3 & 13.2 & 6.37 & 1.21 & 0.13 & T5 & 2 \\
\hline
\end{tabular}

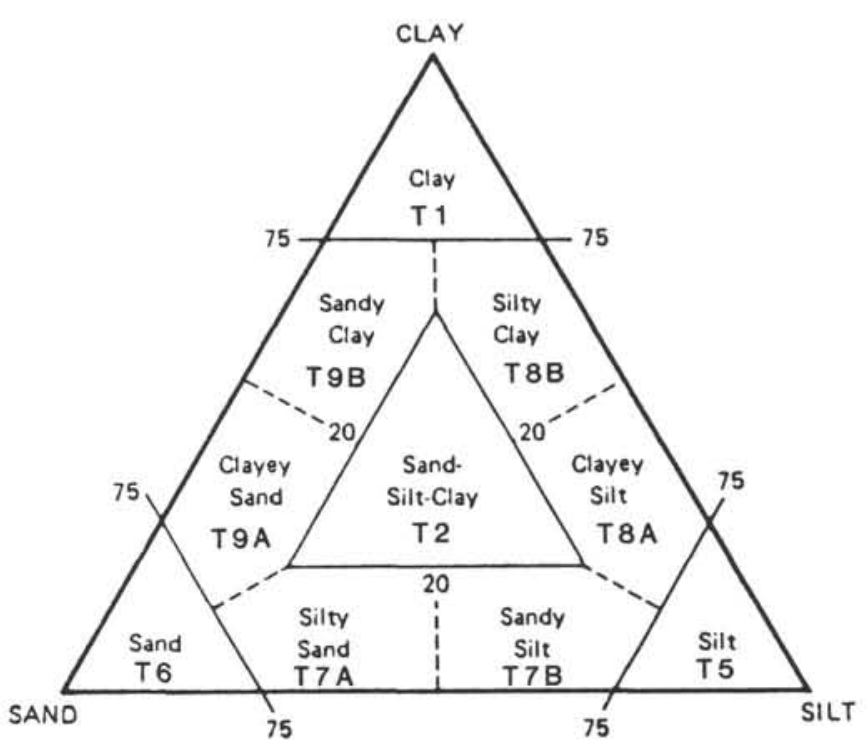

Figure 1. Key to lithologic symbols based on the classification scheme of Shepard (1954).

\section{REFERENCES}

Butters, G., and Wheatley, A. L., 1982. Experience with the Malvern ST18OO Laser Diffraction Particle Sizer. In Stanley-Wood, N. G., and Allen, T. (Eds.), Particle Size Analysis 1981: New York (Wiley Heyden), 425-436.

Folk, R. L., and Ward, W. C., 1957. Brazos River Bar, a study in the significance of grain-size parameters. J. Sediment. Petrol., 27:327.

Jones, K.P.N., McCave, I. N., and Patel, P. D., 1988. A computerinterfaced sedigraph for modal size analysis of fine-grained sediment. Sedimentology, 35:163-172.

McCave, I. N., Bryant, R. J., Cook, H. F., and Coughanowr, C. A., 1986. Evaluation of a laser-diffraction-size analyzer for use with natural sediments. J. Sediment. Petrol., 56:561-564.

Shepard, F. P., 1954. Nomenclature based on sand-silt-clay ratios. J, Sediment. Petrol., 24:151-158.

Singer, J. K., Anderson, J. B., Ledbetter, M. T., McCave, I. N., Jones, K.P.N., and Wright, R., 1988. An assessment of analytical techniques for the size analysis of fine-grained sediments. $J$. Sediment. Petrol., 58:534-543.

Date of initial receipt: 14 July 1989

Date of acceptance: 16 February 1990 Ms 116B-113 